\title{
Superrigidity of Hyperbolic Buildings
}

\author{
Georgios Daskalopoulos \\ Brown University \\ daskal@math . brown .edu \\ Chikako Mese ${ }^{1}$ \\ Johns Hopkins University \\ cmese@math.jhu.edu \\ and \\ Alina Vdovina \\ University of Newcastle \\ Alina.Vdovina@newcastle.ac.uk
}

\begin{abstract}
We prove rank one and higher rank superrigidity for the isometry groups of a class of complexes which includes hyperbolic buildings as a special case. Our method uses harmonic maps to singular spaces.
\end{abstract}

\section{Introduction.}

An H-connected cell complex is a $k$-dimensional locally finite NPC Riemannian cell complex $Y$ with the property that all cells are bounded polyhedra in hyperbolic space $\mathbf{H}^{k}$ and that for any adjacent cells there is an isometrically embedded and totally geodesic copy of $\mathbf{H}^{k}$ containing both cells (for details see next section). This is the hyperbolic analogue of F-connected Euclidean complexes of [GS] and contains hyperbolic buildings as a special case. Recall the following definition from $[\mathrm{M}]$.

Definition 1 Let $\Gamma$ be a discrete group, $Y$ an $N P C$ space and $\operatorname{Isom}(Y)$ the group of isometries of $Y$. A homomorphism $\rho: \Gamma \rightarrow \operatorname{Isom}(Y)$ is called reduced if there is no unbounded closed convex $Z \subset Y, Z \neq Y$ such that $\rho(\gamma) Z$ is at finite Hausdorff distance from $Z$ for all $\gamma \in \Gamma$.

\footnotetext{
${ }^{1}$ supported by research grant NSF DMS-0450083
} 
The main theorem of this paper is to obtain the following superrigidity result for $\mathrm{H}$-connected complexes.

Theorem 2 Let $\tilde{X}=G / K$ be an irreducible symmetric space of noncompact type, other than $S O_{0}(p, 1) / S O(p) \times S O(1), S U_{0}(p, 1) / S(U(p) \times U(1))$. Let $\Gamma$ be a discrete subgroup of $G$ with finite volume quotient and let $\rho: \Gamma \rightarrow$ Isom $(Y)$ a reduced homomorphism, where $Y$ is an H-connected complex. If the rank of $\tilde{X}$ is $\geq 2$ we assume additionally that $\Gamma$ is cocompact. Then $\rho(\Gamma)$ fixes a point of $Y$.

We will deduce the above theorem from its equivalent geometric version.

Theorem 3 Under the same notation and assumptions as in Theorem 2 there exists a finite energy totally geodesic $\rho$-equivariant harmonic map $u$ : $\tilde{X} \rightarrow Y$. In fact, $u$ is constant.

Remarks and Acknowledgements. In the case of symmetric spaces of rank $\geq 2$, Theorem 2 also follows from bounded cohomology considerations (cf. $[\mathrm{MS}]$ and $[\mathrm{MMS}]$ ). We are thankful to Piere Pansu and Nicolas Monod for pointing out those references. The original question for rank 1 superrigidity of hyperbolic buildings was suggested by Mikhail Gromov. In order to keep the conceptual transparency of this article, we defer the bulk of the technical work analyzing the singular set of harmonic maps to the companion article $[\mathrm{DM}]$.

\section{Harmonic maps}

Recall that a metric space $(Y, d)$ is called an $N P C$ space if: (i) The space $(Y, d)$ is a length space. That is, for any two points $P$ and $Q$ in $Y$, there exists a rectifiable curve $c$ so that the length of $c$ is equal to $d(P, Q)$. We call such distance realizing curve a geodesic. (ii) For any three points $P, R, Q \in Y$, let $c:[0, l] \rightarrow Y$ be the arclength parameterized geodesic from $Q$ to $R$ and let $Q_{t}=c(t l)$ for $t \in[0,1]$. Then

$$
d^{2}\left(P, Q_{t}\right) \leq(1-t) d^{2}(P, Q)+t d^{2}(P, R)-t(1-t) d^{2}(Q, R) .
$$


Let $\mathbf{H}^{k}$ denote the hyperbolic space. A convex cell in $\mathbf{H}^{k}$ is a compact intersection of finitely many half spaces in $\mathbf{H}^{k}$. A hyperbolic cell complex $Y$ is a cell complex formed by gluing together convex cells in $\mathbf{H}^{k}$ via isometries of their faces. Throughout the paper we will assume that $Y$ is locally finite and NPC with respect to the induced metric given by

$$
d(x, y)=\inf \{l(\gamma): \gamma \text { is a path from } x \text { to } y\} .
$$

A hyperbolic cell complex is called $H$-connected if for any adjacent cells there is an isometric and totally geodesic embedding $J: \mathbf{H}^{k} \rightarrow Y$ whose image $J\left(\mathbf{H}^{k}\right)$ contains both cells. Such a $J\left(\mathbf{H}^{k}\right)$ will be called a DM (short for differentiable manifold). Notice that H-connected complexes are a special case of DM-complexes considered in [DM]. The main examples of $\mathrm{H}$-connected complexes of interest in this paper are hyperbolic buildings.

We now review the notion of harmonic map. Let $\Omega$ be a smooth bounded $n$-dimensional Riemannian domain and $Y$ an NPC complex. A map $u: \Omega \rightarrow$ $Y$ is said to be an $L^{2}$-map (or that $u \in L^{2}(\Omega, Y)$ ) if for some (and hence all) $P \in Y$, we have

$$
\int_{\Omega} d^{2}(u(x), P) d \mu<\infty .
$$

For $u \in L^{2}(\Omega, Y)$, define the energy density $|\nabla u|^{2}$ as in [GS]. Set

$$
E(u)=\int_{\Omega}|\nabla u|^{2} d \mu
$$

and call a map $u$ of Sobolev class $W^{1,2}(\Omega, Y)$ if $E(u)<\infty$. If $u \in W^{1,2}(\Omega, Y)$, then there exists a well-defined notion of a trace of $u$, denoted $\operatorname{Tr}(u)$, which is an element of $L^{2}(\partial \Omega, Y)$. Two maps $u, v \in W^{1,2}(\Omega, Y)$ have the same trace (i.e. $\operatorname{Tr}(u)=\operatorname{Tr}(v)$ ) if and only if $d(u, v) \in W_{0}^{1,2}(\Omega)$. For details we refer to [KS1]. A map $u: \Omega \rightarrow Y$ is said to be harmonic if it is energy minimizing among all $W^{1,2}$-maps with the same trace.

Similarly there is the notion of equivariant harmonic map. Let $\tilde{X}$ be the universal cover of a complete, finite volume Riemannian manifold $X$, $\Gamma=\pi_{1}(X), Y$ an NPC Riemannian complex and $\rho: \pi_{1}(X) \rightarrow \operatorname{Isom}(Y)$ a homomorphism. Let $u: \tilde{X} \rightarrow Y$ be a $\rho$-equivariant map that is locally of Sobolev class $W^{1,2}$. Since the energy density $|\nabla u|^{2}$ is $\Gamma$-invariant it descends to the quotient and we define

$$
E(u)=\int_{X}|\nabla u|^{2} d \mu .
$$


An equivariant finite energy map $u$ is called harmonic if it is energy minimizing among all finite energy $\rho$-equivariant maps $v: \tilde{X} \rightarrow Y$ which are locally of Sobolev class $W^{1,2}$.

The main regularity result of [GS] and [KS1] is that harmonic maps are locally Lipschitz continuous. The key to Lipschitz regularity is the order function that we shall briefly review. Let $u: \Omega \rightarrow Y$ be a harmonic map. By Section 1.2 of [GS], given $x \in \Omega$ there exists a constant $c>0$ depending only on the $C^{2}$ norm of the metric on $\Omega$ such that

$$
\sigma \mapsto \operatorname{Ord}^{u}(x, \sigma):=e^{c \sigma^{2}} \frac{\sigma E_{x}(\sigma)}{I_{x}(\sigma)}
$$

is non-decreasing for any $x \in \Omega$. In the above, we set

$$
E_{x}(\sigma):=\int_{B_{\sigma}(x)}|\nabla u|^{2} d \mu \text { and } I_{x}(\sigma):=\int_{\partial B_{\sigma}(x)} d^{2}(u, u(x)) d \Sigma(x) .
$$

As a non-increasing limit of continuous functions,

$$
\operatorname{Ord}^{u}(x):=\lim _{\sigma \rightarrow 0} \operatorname{Ord}^{u}(x, \sigma)
$$

is an upper semicontinuous function. By following the proof of Theorem 2.3 in [GS], we see that $\operatorname{Ord}^{u}(x) \geq 1$ (this is equivalent to the Lipschitz property of $u$ ). The value $\operatorname{Ord}^{u}(x)$ is called the order of $u$ at $x$.

The first step in the proof of the main theorem is to show the existence of an equivariant harmonic map.

Lemma 4 Under the same assumptions as in Theorems 2 and 3 there exists a finite energy $\rho$-equivariant harmonic map $u: \tilde{X} \rightarrow Y$.

Proof. As in [GS] Lemma 8.1 in the rank one case, there is a finite energy equivariant map $u: \tilde{X} \rightarrow Y$. In the higher rank case, this is automatically satisfied by the assumption of cocompactness. Now since the property of $\rho$ being reduced in particular implies that $\rho(\Gamma)$ doesn't fix a point at infinity in $Y$, we obtain as in [GS] Theorem 7.1 that $u$ can be deformed to a finite energy equivariant harmonic map. Q.E.D. 


\section{The singular set}

For $Y$ a $k$-dimensional $\mathrm{H}$-connected complex and a point $P \in Y$, let $T_{P} Y$ denote the (Alexandrov) tangent cone of $Y$ at $P$. As explained in [DM], $T_{P} Y$ is an unbounded F-connected Euclidean cell complex obtained by taking the tangent spaces to all the DM's passing through $P$ with the appropriate identifications. Furthermore, the exponential map

$$
\exp _{P}^{Y}: B_{r}(0) \subset T_{P} Y \rightarrow B_{r}(P) \subset Y
$$

is defined by piecing together the exponential maps of all the DM's containing $P$.

Now let $u: \Omega \rightarrow Y$ be a harmonic map. Recall from [DM] that a point $x_{0} \in \Omega$ is called a regular point if $\operatorname{Ord}^{u}\left(x_{0}\right)=1$ and there exists $\sigma_{0}>0$ such that

$$
u\left(B_{\sigma_{0}}\left(x_{0}\right)\right) \subset \exp _{u\left(x_{0}\right)}^{Y}\left(X_{0}\right),
$$

where $X_{0} \subset T_{u\left(x_{0}\right)} Y$ is isometric to $\mathbf{R}^{k}$. In particular, $x_{0}$ has a neighborhood mapping into a DM. A point $x_{0} \in \Omega$ is called a singular point if it is not a regular point. Denote the set of regular points by $\mathcal{R}(u)$ and the set of singular points by $\mathcal{S}(u)$. The main result of $[\mathrm{DM}]$ is the following theorem

Theorem 5 (cf. $[D M]$ ) Let $\Omega$ be an n-dimensional Riemannian domain, $Y$ a $k$-dimensional $H$-connected complex and $u: \Omega \rightarrow Y$ a harmonic map. Then the singular set $\mathcal{S}(u)$ of $u$ has Hausdorff co-dimension 2 in $\Omega$; i.e.

$$
\operatorname{dim}_{\mathcal{H}}(\mathcal{S}(u)) \leq n-2
$$

Following $[\mathrm{DM}]$ we will stratify the singular set further into the following subsets. Set

$$
\mathcal{S}_{0}(u)=\left\{x_{0} \in \Omega: \operatorname{Ord}^{u}\left(x_{0}\right)>1\right\},
$$

$k_{0}:=\min \{n, k\}$ and $\mathcal{S}_{j}(u)=\emptyset$ if $j \geq k_{0}+1$ or $j \leq-1$. For $j=1, \ldots, k_{0}$, we define $\mathcal{S}_{j}(u)$ inductively as follows. Having defined $\mathcal{S}_{m}(u)$ for $m=j+1, j+$ $2, \ldots$, define $\mathcal{S}_{j}(u)$ to be the set of points

$$
x_{0} \in \mathcal{S}(u) \backslash\left(\bigcup_{m=j+1}^{k_{0}} \mathcal{S}_{m}(u) \cup \mathcal{S}_{0}(u)\right)
$$


with the property that there exists $\sigma_{0}>0$ such that

$$
u\left(B_{\sigma_{0}}\left(x_{0}\right)\right) \subset \exp _{u\left(x_{0}\right)}^{Y}\left(X_{0}\right)
$$

where $X_{0} \subset T_{u\left(x_{0}\right)} Y$ is isometric to $\mathbf{R}^{j} \times Y_{2}^{k-j}$ and $Y_{2}$ is $(k-j)$-dimensional F-connected complex. Set

$$
\mathcal{S}_{m}^{-}(u)=\bigcup_{j=0}^{m} \mathcal{S}_{j}(u) \text { and } \mathcal{S}_{m}^{+}(u)=\bigcup_{j=m}^{k} \mathcal{S}_{j}(u) \text {. }
$$

It was shown in $[\mathrm{DM}]$ that

Lemma 6 The sets $\mathcal{S}_{0}(u), \mathcal{S}_{1}(u), \ldots, \mathcal{S}_{k_{0}-1}(u), \mathcal{S}_{k_{0}}(u)$ form a partition of $\mathcal{S}(u)$. Furthermore, the sets $\mathcal{R}(u), \mathcal{R}(u) \cup \mathcal{S}_{m}^{+}(u)$ are open and the sets $\mathcal{S}_{m}^{-}(u)$ are closed.

Let $\left(0, P_{0}\right)$ be the vertex of $\mathbf{R}^{j} \times Y_{2}^{k-j}$ (i.e. the point corresponding to the vertex of $T_{u\left(x_{0}\right)} Y$ via the isometry between $\mathbf{R}^{j} \times Y_{2}^{k-j}$ and $X_{0} \subset$ $\left.T_{u\left(x_{0}\right)} Y\right)$. Define a metric $G$ on $\mathbf{R}^{j} \times Y_{2}^{k-j}$ by pulling back the metric on $Y$ via the exponential map in a neighborhood of $\left(0, P_{0}\right)$. When studying local properties of the harmonic map $u: \Omega \rightarrow Y$ at $x_{0} \in \Omega$, we may assume that $u$ maps $B_{\sigma_{0}}\left(x_{0}\right) \subset \Omega$ into $\left(\mathbf{R}^{j} \times Y_{2}^{k-j}, G\right)$, where $j \geq 0$. We need the following two lemmas.

Lemma 7 Let $u=\left(u^{1}, u^{2}\right):\left(B_{\sigma_{0}}\left(x_{0}\right), g\right) \rightarrow\left(\mathbf{R}^{j} \times Y_{2}^{k-j}, G\right)$ be the harmonic map with $u\left(x_{0}\right)=\left(0, P_{0}\right)$ given above and $j>0$. If we write $u^{1}=\left(u_{I}^{1}\right)_{I=1, \ldots, j}$ : $B_{\sigma_{0}}\left(x_{0}\right) \rightarrow \mathbf{R}^{j}$, then $u_{I}^{1} \in W_{\text {loc }}^{2, p}\left(B_{\frac{\sigma_{0}}{2}}\left(x_{0}\right)\right)$ for any $p \in(0, \infty)$ and any $I=$ $1, \ldots, j$. In particular, $\left|\nabla u^{1}\right|$ is continuous in $B_{\frac{\sigma_{0}}{2}}\left(x_{0}\right)$.

Proof. Let $R>0$ such that $u\left(B_{\sigma_{0}}\left(x_{0}\right)\right)$ is contained in a ball $B_{R}$ of radius $R$ about $\left(0, P_{0}\right)$. For any DM $M$ of $\left(\mathbf{R}^{j} \times Y_{2}^{k-j}, G\right)$, extend coordinates on $\mathbf{R}^{j}$ to define coordinates of $B_{2 R} \cap M$. Since $\mathbf{R}^{j} \times Y_{2}^{k-j}$ is a locally finite complex there exist a finite number of distinct DM's $M_{1}, \ldots, M_{L}$ contained in $\mathbf{R}^{j} \times Y_{2}^{k-j}$. Define

$$
C_{1}:=\max _{l=1, \ldots, L} \max _{i, r, s=1, \ldots, k} \sup _{M_{l} \cap \bar{B}_{R}}\left|{ }^{M_{l}} \Gamma_{r s}^{i}\right|
$$


where ${ }^{M_{l}} \Gamma_{r s}^{i}$ is the Christoffel symbols of $M_{l}$ with respect to the coordinates in $B_{2 R} \cap M_{l}$ as above. Let $u_{i}^{M_{l}}$ for $i=1, \ldots, k$ denote the $i$ th coordinate function. Here, we emphasize that

$$
u_{I}^{M_{l}}=u_{I}^{1} \text { for } I=1, \ldots, j
$$

by the construction above. In particular, note that $u_{I}^{M_{l}}=u_{I}^{M_{l^{\prime}}}$ for any $l, l^{\prime}=1, \ldots, L$. Define

$$
C_{2}:=\max _{l=1, \ldots, L} \max _{i=1, \ldots, k} \max _{\alpha=1, \ldots, n}\left|\frac{\partial u_{i}^{M_{l}}}{\partial x^{\alpha}}\right|_{L^{\infty}\left(B_{\frac{3 \sigma_{0}}{4}}\left(x_{0}\right) \cap u^{-1}\left(M_{l}\right)\right)} .
$$

For $x \in \mathcal{R}(u)$, let $M_{l}$ be the DM containing $u\left(B_{\delta}(x)\right)$ for some $\delta>0$. We have the harmonic map equation

$$
\triangle u_{i}^{M_{l}}=-g^{\alpha \beta M_{l}} \Gamma_{r s}^{i}(u) \frac{\partial u_{r}^{M_{l}}}{\partial x^{\alpha}} \frac{\partial u_{s}^{M_{l}}}{\partial x^{\beta}}
$$

in $B_{\delta}(x)$. Thus, for $\delta$ sufficiently small,

$$
\left|\triangle u_{I}^{1}\right|_{L^{\infty}\left(B_{\delta}(x)\right)}=\left|\triangle u_{I}^{M_{l}}\right|_{L^{\infty}\left(B_{\delta}(x)\right)} \leq C C_{1} C_{2}^{2} \forall I=1, \ldots, j,
$$

where the constant $C$ depends only on the dimension $n$ and the metric $g$ of the domain. Since $\operatorname{dim}_{\mathcal{H}}\left(B_{\frac{3 \sigma_{0}}{4}}\left(x_{0}\right) \backslash \mathcal{R}(u)\right) \leq n-2$, we see that the inequality (3) implies $\triangle u_{I}^{1} \in L^{p}\left(B_{\frac{3 \sigma_{0}}{4}}\left(x_{0}\right)\right)$ which in turn implies $u_{I}^{1} \in W^{2, p}\left(B_{\frac{\sigma_{0}}{2}}\left(x_{0}\right)\right)$. Q.E.D.

We now prove

Lemma 8 Let $\Omega$ be an n-dimensional Riemannian domain, $Y$ a $k$-dimensional $H$-connected complex and $u: \Omega \rightarrow Y$ a harmonic map. For any compact subdomain $\Omega_{1}$ of $\Omega$, there exists a sequence of smooth functions $\left\{\psi_{i}\right\}$ with $\psi_{i} \equiv 0$ in a neighborhood of $\mathcal{S}(u) \cap \overline{\Omega_{1}}, 0 \leq \psi_{i} \leq 1$ and $\psi_{i} \rightarrow 1$ for all $x \in \Omega \backslash\left(\mathcal{S}(u) \cap \overline{\Omega_{1}}\right)$ such that

$$
\lim _{i \rightarrow \infty} \int_{\Omega}|\nabla \nabla u|\left|\nabla \psi_{i}\right| d \mu=0
$$


Proof. The proof of the Lemma follows by induction from the following

ClaIm. Assume that given any subdomain $\Omega_{1}^{\prime}$ compactly contained in $\Omega \backslash \mathcal{S}_{j}^{-}(u)$, there exists a sequence of smooth functions $\left\{\hat{\psi}_{i}\right\}$ with $\hat{\psi}_{i} \equiv 0$ in a neighborhood of $\mathcal{S}_{j+1}^{+}(u) \cap \overline{\Omega_{1}^{\prime}}, 0 \leq \hat{\psi}_{i} \leq 1, \hat{\psi}_{i} \rightarrow 1$ for all $x \in \Omega \backslash\left(\mathcal{S}_{j+1}^{+}(u) \cap \overline{\Omega_{1}^{\prime}}\right)$ such that

$$
\lim _{i \rightarrow \infty} \int_{\Omega}\left|\nabla \hat{\psi}_{i}\right| d \mu=0
$$

and

$$
\lim _{i \rightarrow \infty} \int_{\Omega}|\nabla \nabla u|\left|\nabla \hat{\psi}_{i}\right| d \mu=0 .
$$

Then given any subdomain $\Omega_{1}$ compactly contained in $\Omega \backslash \mathcal{S}_{j-1}^{-}(u)$, there exists a sequence of smooth functions $\left\{\psi_{i}\right\}$ with $\psi_{i} \equiv 0$ in a neighborhood of $\mathcal{S}_{j}^{+}(u) \cap$ $\overline{\Omega_{1}}, 0 \leq \psi_{i} \leq 1, \psi_{i} \rightarrow 1$ for all $x \in \Omega \backslash\left(\mathcal{S}_{j}^{+}(u) \cap \overline{\Omega_{1}}\right)$ such that

$$
\lim _{i \rightarrow \infty} \int_{\Omega}\left|\nabla \psi_{i}\right| d \mu=0
$$

and

$$
\lim _{i \rightarrow \infty} \int_{\Omega}|\nabla \nabla u|\left|\nabla \psi_{i}\right| d \mu=0
$$

We now prove the claim. For a subdomain $\Omega_{1}$ compactly contained in $\Omega \backslash \mathcal{S}_{j-1}^{-}(u)$, let $\Omega_{2}$ be such that $\Omega_{1} \subset \subset \Omega_{2} \subset \subset \Omega \backslash \mathcal{S}_{j-1}^{-}(u)$. Without the loss of generality, we can assume that $u=\left(u^{1}, u^{2}\right): \Omega_{2} \rightarrow\left(\mathbf{R}^{j} \times Y_{2}^{k-j}, G\right)$ and that $\nabla u^{1} \in W^{1, p}\left(\Omega_{2}\right) \cap C^{0}\left(\overline{\Omega_{2}}\right)$ for any $p>0$ by Lemma 7 . Furthermore, we claim that $\left|\nabla u^{1}\right| \neq 0$ in $S_{j}(u) \cap \overline{\Omega_{1}}$. Indeed, if $\left|\nabla u^{1}\right|(x)=0$ for some $x \in S_{j}(u) \cap \overline{\Omega_{1}}$, then the Gap theorem in $[\mathrm{DM}]$ implies that $\left|\nabla u^{2}\right|(x)=0$ and therefore also $|\nabla u|(x)=0$, contradicting the fact that $x$ is a point of order 1. In particular, this means that there exists a neighborhood $\mathcal{N} \subset \Omega_{2}$ of $\mathcal{S}_{j}(u) \cap \overline{\Omega_{1}}$ and a constant $\delta_{0}$ such that

$$
\left|\nabla u^{1}\right| \geq \delta_{0}>0 \text { on } \mathcal{N} \text {. }
$$

Below, we will use $C$ to denote any generic constant which only depends on $\delta_{0}$, the dimension of $n$ of $\Omega$ and the Lipschitz constant of $u$. For $d \in(n-2, n)$ to be chosen later, fix a finite covering $\left\{B_{r_{J}}\left(x_{J}\right): J=1, \ldots, l\right\}$ of the compact set $\mathcal{S}_{j}(u) \cap \overline{\Omega_{1}}$ satisfying

$$
\sum_{J=1}^{l} r_{J}^{d} \leq \epsilon
$$


We also assume

$$
B_{3 r_{J}}\left(x_{J}\right) \subset \mathcal{N}
$$

which is true if $\epsilon>0$ is small and that $x_{J} \in \mathcal{S}_{j}(u) \cap \overline{\Omega_{1}}$. Let $\varphi_{J}$ be a smooth function which is zero on $B_{r_{J}}\left(x_{J}\right)$ and identically one on $\Omega \backslash B_{2 r_{J}}\left(x_{J}\right)$ such that $\left|\nabla \varphi_{J}\right| \leq C r_{J}^{-1},\left|\nabla \nabla \varphi_{J}\right| \leq C r_{J}^{-2}$ and $\left|\nabla \nabla \nabla \varphi_{J}\right| \leq C r_{J}^{-3}$. If $\varphi$ is defined by

$$
\varphi=\min \left\{\varphi_{J}: J=1, \ldots, l\right\},
$$

then $\varphi \equiv 0$ in a neighborhood of $\mathcal{S}_{j}(u) \cap \overline{\Omega_{1}}$ and $\varphi \equiv 1$ on $\Omega_{1} \backslash \cup_{J=1}^{l} B_{2 r_{J}}\left(x_{J}\right)$. Let

$$
\Omega_{1}^{\prime}=\Omega_{1} \backslash \cup_{J=1}^{l} B_{r_{J}}\left(x_{J}\right)
$$

and let $\left\{\hat{\psi}_{i}\right\}$ be as in the inductive hypothesis. Now let $\psi_{0}=\varphi^{2} \hat{\psi}_{i}$. Then for $i$ sufficiently large, we have

$$
\begin{aligned}
\int_{\Omega}\left|\nabla \psi_{0}\right| d \mu & \leq 2 \int_{\Omega} \varphi \hat{\psi}_{i}|\nabla \varphi| d \mu+\varphi^{2}\left|\nabla \hat{\psi}_{i}\right| d \mu \\
& \leq C \sum_{J=1}^{l} r_{J}^{n-1}+\int_{\Omega}\left|\nabla \hat{\psi}_{i}\right| d \mu \leq C \epsilon+\epsilon
\end{aligned}
$$

Furthermore, we have

$$
\begin{aligned}
\int_{\Omega}|\nabla \nabla u|\left|\nabla \psi_{0}\right| d \mu & =2 \int_{\Omega} \varphi \hat{\psi}_{i}|\nabla \nabla u||\nabla \varphi| d \mu+\int_{\Omega} \varphi^{2}|\nabla \nabla u|\left|\nabla \hat{\psi}_{i}\right| d \mu \\
& \leq 2 \int_{\Omega} \varphi \hat{\psi}_{i}|\nabla \nabla u||\nabla \varphi| d \mu+\int_{\Omega}|\nabla \nabla u|\left|\nabla \hat{\psi}_{i}\right| d \mu .
\end{aligned}
$$

For $\delta \in(0,1)$ to be chosen later, we write the first term as

$$
\begin{aligned}
& 2 \int_{\Omega} \varphi \hat{\psi}_{i}|\nabla \nabla u||\nabla \varphi| d \mu \\
& \leq\left.2 \int_{\cup_{J=1}^{l} B_{2 r_{J}\left(x_{J}\right)} \varphi \hat{\psi}_{i}|\nabla \nabla u||\nabla \varphi| d \mu}|\nabla \nabla u|^{2}|\nabla u|^{-1} \varphi^{2} \hat{\psi}_{i}|\nabla \varphi|^{\delta} d \mu\right)^{1 / 2} \\
& \leq 2\left(\int_{\cup_{J=1}^{l} B_{2 r_{J}\left(x_{J}\right)} \mid \nabla}|\nabla u||\nabla \varphi|^{2-\delta} \hat{\psi}_{i} d \mu\right)^{1 / 2} \\
& \times\left(\int_{\cup_{J=1}^{l} B_{2 r_{J}}\left(x_{J}\right)}|\nabla u|^{2}|\nabla u|^{-1} \varphi^{2} \hat{\psi}_{i}|\nabla \varphi|^{\delta} d \mu\right)^{1 / 2}\left(C \sum_{J=1}^{l} r_{J}^{n-2+\delta}\right)^{1 / 2} .
\end{aligned}
$$


Let $\rho_{J}$ be a Lipchitz function which is identically one on $B_{2 r_{J}}\left(x_{J}\right)$ and identically zero on $\Omega \backslash B_{3 r_{J}}\left(x_{J}\right)$ with $\left|\nabla \rho_{J}\right| \leq C r_{J}^{-1}$ and $\left|\nabla \nabla \rho_{J}\right| \leq C r_{J}^{-2}$. Define

$$
\rho=\max \left\{\rho_{J}: J=1, \ldots, l\right\} .
$$

As in [GS] Theorem 6.4 on $\mathcal{R}(u)$, we will use the pointwise inequalities

$$
\frac{1}{2} \triangle|\nabla u|^{2} \geq|\nabla \nabla u|^{2}-c|\nabla u|^{2} \text { and }\left(1-\epsilon_{n}\right)|\nabla \nabla u|^{2} \geq\left.|\nabla| \nabla u\right|^{2}
$$

with constant $\epsilon_{n}$ depending only on $n$ which combine to imply

$$
\epsilon_{n}|\nabla \nabla u|^{2}|\nabla u|^{-1} \leq \triangle|\nabla u|+c|\nabla u| \text { on } \mathcal{R}(u) .
$$

Since $\varphi^{2} \rho^{2} \hat{\psi}_{i} \equiv 0$ in a neighborhood of $\mathcal{S}_{j}^{+}(u) \cap\left(\cup_{J=1}^{l} B_{2 r_{J}}\left(x_{J}\right)\right)$, we have that

$$
\begin{aligned}
& \int_{\cup_{J=1}^{l} B_{2 r_{J}}\left(x_{J}\right)}|\nabla \nabla u|^{2}|\nabla u|^{-1} \varphi^{2} \hat{\psi}_{i}|\nabla \varphi|^{\delta} d \mu \\
& \quad \leq \int_{\Omega}|\nabla \nabla u|^{2}|\nabla u|^{-1} \varphi^{2} \rho^{2} \hat{\psi}_{i}|\nabla \varphi|^{\delta} d \mu \\
& \leq \frac{1}{\epsilon_{n}} \int_{\Omega} \Delta|\nabla u| \varphi^{2} \rho^{2} \hat{\psi}_{i}|\nabla \varphi|^{\delta} d \mu+\frac{c}{\epsilon_{n}} \int_{\Omega}|\nabla u| \varphi^{2} \rho^{2} \hat{\psi}_{i}|\nabla \varphi|^{\delta} d \mu .
\end{aligned}
$$

The second term of the right-hand side has the estimate

$$
\frac{c}{\epsilon_{n}} \int_{\Omega}|\nabla u| \varphi^{2} \rho^{2} \hat{\psi}_{i}|\nabla \varphi|^{\delta} d \mu \leq C \int_{\cup_{J=1}^{l} B_{2 r_{J}}\left(x_{J}\right)}|\nabla \varphi|^{\delta} \leq C \sum_{J=1}^{l} r_{J}^{n-\delta} .
$$

The first term can be rewritten

$$
\begin{aligned}
& \frac{1}{\epsilon_{n}} \int_{\Omega}|\nabla u| \triangle\left(\varphi^{2} \rho^{2} \hat{\psi}_{i}|\nabla \varphi|^{\delta}\right) d \mu \\
& \leq \quad C \int_{\Omega} \hat{\psi}_{i}|\nabla u| \triangle\left(\varphi^{2} \rho^{2}|\nabla \varphi|^{\delta}\right) d \mu+C \int_{\Omega} \varphi^{2} \rho^{2}|\nabla \varphi|^{\delta}|\nabla u| \triangle \hat{\psi}_{i} d \mu \\
& \quad+C \int_{\Omega}|\nabla u|<\nabla\left(\varphi^{2} \rho^{2}|\nabla \varphi|^{\delta}\right) \cdot \nabla \hat{\psi}_{i}>d \mu \\
& =(a)+(b)+(c) .
\end{aligned}
$$

By the mean value theorem,

$$
\frac{\left(\left|\nabla u^{1}\right|^{2}+s\right)^{\frac{1}{2}}-\left|\nabla u^{1}\right|}{s}=\frac{1}{2}\left(\left|\nabla u^{1}\right|^{2}+c\right)^{-\frac{1}{2}}
$$


for some $c \in(0, s)$. Letting $s=\left|\nabla u^{2}\right|^{2}+2<\nabla u^{1}, \nabla u^{2}>$, we have

$$
|\nabla u|=\left|\nabla u^{1}\right|+\frac{1}{2}\left(\left|\nabla u^{1}\right|^{2}+c\right)^{-\frac{1}{2}}\left(\left|\nabla u^{2}\right|^{2}+2<\nabla u^{1}, \nabla u^{2}>\right) .
$$

Thus,

$$
\begin{aligned}
(a)= & C \int_{\Omega} \hat{\psi}_{i}|\nabla u| \triangle\left(\varphi^{2} \rho^{2}|\nabla \varphi|^{\delta}\right) d \mu \\
= & C \int_{\Omega} \hat{\psi}_{i}\left|\nabla u^{1}\right| \triangle\left(\varphi^{2} \rho^{2}|\nabla \varphi|^{\delta}\right) d \mu \\
& +C \int_{\Omega} \frac{1}{2} \hat{\psi}_{i}\left(\left|\nabla u^{1}\right|^{2}+c\right)^{-\frac{1}{2}}\left(\left|\nabla u^{2}\right|^{2}+2<\nabla u^{1}, \nabla u^{2}>\right) \triangle\left(\varphi^{2} \rho^{2}|\nabla \varphi|^{\delta}\right) d \mu \\
= & (a)_{1}+(a)_{2} .
\end{aligned}
$$

For $p$ and $q$ with $\frac{1}{p}+\frac{1}{q}=1$ to be chosen later, we have

$$
\begin{aligned}
(a)_{1} & =C \int_{\Omega} \hat{\psi}_{i}\left|\nabla u^{1}\right| \triangle\left(\varphi^{2} \rho^{2}|\nabla \varphi|^{\delta}\right) d \mu \\
& =-C \int_{\Omega}<\nabla\left(\hat{\psi}_{i}\left|\nabla u^{1}\right|\right), \nabla\left(\varphi^{2} \rho^{2}|\nabla \varphi|^{\delta}\right)>d \mu \\
& \leq C \int_{\Omega}\left|\nabla \hat{\psi}_{i}\right|\left|\nabla u^{1}\right|\left|\nabla\left(\varphi^{2} \rho^{2}|\nabla \varphi|^{\delta}\right)\right| d \mu+C \int_{\Omega}|\nabla| \nabla u^{1}||\left|\nabla\left(\varphi^{2} \rho^{2}|\nabla \varphi|^{\delta}\right)\right| d \mu \\
& \leq C \sum_{J=1}^{l} r_{J}^{-(1+\delta)} \int_{\Omega}\left|\nabla \hat{\psi}_{i}\right|+C\left(\left.\int_{\Omega}|\nabla| \nabla u^{1}\right|^{p} d \mu\right)^{1 / p}\left(\int_{\Omega}\left|\nabla\left(\varphi^{2} \rho^{2}|\nabla \varphi|^{\delta}\right)\right|^{q} d \mu\right)^{1 / q} \\
& \leq C \sum_{J=1}^{l} r_{J}^{-(1+\delta)} \int_{\Omega}\left|\nabla \hat{\psi}_{i}\right|+C\left(\left.\int_{\Omega}|\nabla| \nabla u^{1}\right|^{p} d \mu\right)^{1 / p}\left(\sum_{J=1}^{l} r_{J}^{n-(1+\delta) q}\right)^{1 / q} .
\end{aligned}
$$

Furthermore, using (4) and (6), we have

$$
\begin{aligned}
(a)_{2} & =C \int_{\Omega} \frac{1}{2} \hat{\psi}_{i}\left(\left|\nabla u^{1}\right|^{2}+c\right)^{-\frac{1}{2}}\left(\left|\nabla u^{2}\right|^{2}+2<\nabla u^{1}, \nabla u^{2}>\right) \triangle\left(\varphi^{2} \rho^{2}|\nabla \varphi|^{\delta}\right) d \mu \\
& =C \int_{\Omega}\left(\left|\nabla u^{2}\right|^{2}+2<\nabla u^{1}, \nabla u^{2}>\right) \triangle\left(\varphi^{2} \rho^{2}|\nabla \varphi|^{\delta}\right) d \mu \\
& =C \sum_{J=1}^{l} r_{J}^{-(2+\delta)}\left(\int_{\Omega}\left|\nabla u^{2}\right|^{2} d \mu+\int_{B_{r_{J}}\left(x_{J}\right)} 2<\nabla u^{1}, \nabla u^{2}>d \mu\right) \\
& \leq C \sum_{J=1}^{l} r_{J}^{-(2+\delta)}\left(\int_{\Omega}\left|\nabla u^{2}\right|^{2} d \mu+2\left(\int_{\Omega}\left|\nabla u^{1}\right|^{2} d \mu\right)^{1 / 2}\left(\int_{\Omega}\left|\nabla u^{2}\right|^{2} d \mu\right)^{1 / 2}\right) \\
& \leq C \sum_{J=1}^{l} r_{J}^{n-(2+\delta)+\frac{\epsilon a p}{2}}
\end{aligned}
$$


where $\epsilon_{\text {gap }}$ is the order gap for approximately harmonic maps into $Y_{2}^{k-j}$ as described in $[\mathrm{DM}]$. Additionally,

$$
\begin{aligned}
(b) & =C \int_{\Omega} \varphi^{2} \rho^{2}|\nabla \varphi|^{\delta}|\nabla u| \triangle \hat{\psi}_{i} d \mu \\
& \leq C \int_{\Omega}\left|\nabla\left(\varphi^{2} \rho^{2}|\nabla \varphi|^{\delta}\right)\right||\nabla u|\left|\nabla \hat{\psi}_{i}\right| d \mu+C \int_{\Omega} \varphi^{2} \rho^{2}|\nabla \varphi|^{\delta}|\nabla| \nabla u||\left|\nabla \hat{\psi}_{i}\right| d \mu \\
& \leq C \sum_{J=1}^{l} r_{J}^{-(1+\delta)} \int_{\Omega}\left|\nabla \hat{\psi}_{i}\right| d \mu+C \sum_{J=1}^{l} r_{J}^{-\delta} \int_{\Omega}|\nabla \nabla u|\left|\nabla \hat{\psi}_{i}\right| d \mu
\end{aligned}
$$

and

$$
(c)=C \int_{\Omega}|\nabla u|<\nabla\left(\varphi^{2} \rho^{2}|\nabla \varphi|^{\delta}\right) \cdot \nabla \hat{\psi}_{i}>d \mu \leq C \sum_{J=1}^{l} r_{J}^{-(1+\delta)} \int_{\Omega}\left|\nabla \hat{\psi}_{i}\right| d \mu .
$$

Thus, we obtain

$$
\begin{aligned}
& \frac{1}{\epsilon_{n}} \int_{\Omega}|\nabla u| \triangle\left(\varphi^{2} \rho^{2} \hat{\psi}_{i}|\nabla \varphi|^{\delta}\right) d \mu \\
& \leq C \sum_{J=1}^{l} r_{J}^{-(1+\delta)} \int_{\Omega}\left|\nabla \hat{\psi}_{i}\right|+C\left(\left.\int_{\Omega}|\nabla| \nabla u^{1}\right|^{p} d \mu\right)^{1 / p}\left(\sum_{J=1}^{l} r_{J}^{n-(1+\delta) q}\right)^{1 / q} \\
& \quad+C \sum_{J=1}^{l} r_{J}^{n-(2+\delta)+\frac{\epsilon g a p}{2}}+C \sum_{J=1}^{l} r_{J}^{-\delta} \int_{\Omega}|\nabla \nabla u|\left|\nabla \hat{\psi}_{i}\right| d \mu .
\end{aligned}
$$

Combining all the above estimates, we obtain

$$
\begin{aligned}
& \int_{\Omega}|\nabla \nabla u|\left|\nabla \psi_{0}\right| d \mu \\
& \leq 2\left(C \sum_{J=1}^{l} r_{J}^{-(1+\delta)} \int_{\Omega}\left|\nabla \hat{\psi}_{i}\right|+C\left(\int_{\Omega}|\nabla| \nabla u^{1}||^{p} d \mu\right)^{1 / p}\left(\sum_{J=1}^{l} r_{J}^{n-(1+\delta) q}\right)^{\frac{1}{q}}\right. \\
& \left.\quad+C \sum_{J=1}^{l} r^{n-(2+\delta)+\frac{\epsilon g a p}{2}}+C \sum_{J=1}^{l} r_{J}^{-\delta} \int_{\Omega}|\nabla \nabla u|\left|\nabla \hat{\psi}_{i}\right| d \mu+\sum_{J=1}^{l} r_{J}^{n-\delta}\right)^{1 / 2} \\
& \quad\left(C \sum_{J=1}^{l} r_{J}^{n-2+\delta}\right)^{1 / 2}+\int_{\Omega}|\nabla \nabla u|\left|\nabla \hat{\psi}_{i}\right| d \mu .
\end{aligned}
$$

First we choose $0<\delta<\frac{\epsilon_{g a p}}{2}$ and then choose $d$ in (5) such that $d \in(n-2, n-$ $\left.(2+\delta)+\frac{\epsilon_{\text {gap }}}{2}\right)$ and $d<n-2+\delta$. Then choose $q>1$ such that $n-(1+\delta) q>d$ 
and $p$ such that $\frac{1}{p}+\frac{1}{q}=1$. Last fix $i$ sufficiently large such that

$$
\sum_{J=1}^{l} r_{J}^{-(1+\delta)} \int_{\Omega}\left|\nabla \hat{\psi}_{i}\right| d \mu, \sum_{J=1}^{l} r_{J}^{-\delta} \int_{\Omega}|\nabla \nabla u|\left|\nabla \hat{\psi}_{i}\right| d \mu<\epsilon .
$$

We then have

$$
\begin{aligned}
& \int_{\Omega}|\nabla \nabla u|\left|\nabla \psi_{0}\right| d \mu \\
& \quad \leq 2\left(C \epsilon+C\left(\left.\int_{\Omega}|\nabla| \nabla u^{1}\right|^{p} d \mu\right)^{1 / p} \epsilon+3 C \epsilon\right)^{1 / 2}(C \epsilon)^{1 / 2}+\epsilon
\end{aligned}
$$

Finally, note that $\psi_{i} \equiv 0$ in a neighborhood $\mathcal{S}_{j+1}(u) \cap\left(\overline{\Omega_{1}} \backslash \cup_{J=1}^{l} B_{r_{J}}\left(x_{J}\right)\right.$, $\varphi \equiv 0$ in $\cup B_{r_{J}}\left(x_{J}\right)$ and hence $\psi_{0} \equiv 0$ in a neighborhood of $\mathcal{S}_{j}(u) \cap \overline{\Omega_{1}}$. Since $\epsilon>0$ is arbitrary, this proves the claim and finishes the proof of the lemma. Q.E.D.

\section{Proof of the main theorem.}

We first start with the following

Lemma 9 Let $\tilde{X}=G / K$ be the quaternionic hyperbolic space or the Cayley plane, $\Gamma$ a discrete subgroup of $G$ and $\rho: \Gamma \rightarrow I \operatorname{som}(Y)$. Assume $u: \tilde{X} \rightarrow Y$ is a finite energy $\rho$-equivariant harmonic map where $Y$ is a $k$-dimensional $H$-connected complex. Then $D^{*}(d u \wedge \omega)=0$ in a neighborhood of a regular point. In particular, $u$ is totally geodesic (i.e. $\nabla d u=0$ ) in a neighborhood of a regular point.

Proof. The proof is very similar to [GS] Theorem 7.2 so we will only sketch the argument. Let $\omega$ be either the Quaternionic Kähler 4-form or the Cayley 8-form and $x_{0} \in \tilde{X}$ be a regular point. As in [GS], we will work on the quotient $X=\tilde{X} / \Gamma$. Fix $R>0$ and a nonnegative smooth function $\rho$ which is identically one in $B_{R}\left(x_{0}\right)$ and zero outside $B_{2 R}\left(x_{0}\right)$ with $|\nabla \rho| \leq 2 R^{-1}$. Let $\psi$ be a nonnegative smooth function vanishing in a small neighborhood of $\mathcal{S}(u) \cap \overline{B_{2 R}\left(x_{0}\right)}$. By Stokes theorem we obtain

$$
\begin{aligned}
0 & =\int_{X}\left\langle D\left(\psi \rho^{2} *(d u \wedge \omega)\right), D *(d u \wedge * \omega)\right\rangle d \mu \\
& +(-1)^{m-p-1} \int_{X} \psi \rho^{2}\left\langle *(d u \wedge \omega), D^{2} *(d u \wedge * \omega)\right\rangle d \mu .
\end{aligned}
$$


Combining the above with the Corlette formula

$$
* D *(d u \wedge \omega)=(-1)^{m-1} D *(d u \wedge * \omega)
$$

in $\mathcal{R}(u)$ gives

$$
\begin{aligned}
0 & =\int_{X}\left\langle d\left(\psi \rho^{2}\right) \wedge *(d u \wedge \omega), * D *(d u \wedge \omega)\right\rangle d \mu+\int_{X} \psi \rho^{2}|D *(\omega \wedge d u)|^{2} d \mu \\
& +(-1)^{p} \int_{X} \psi \rho^{2}\left\langle *(d u \wedge \omega), D^{2} *(d u \wedge * \omega)\right\rangle d \mu .
\end{aligned}
$$

By $[\mathrm{C}]$ the last two quantities are nonnegative. Hence for any $\epsilon>0$, we have

$$
\begin{aligned}
0 & \leq \int_{B_{R}\left(x_{0}\right)} \psi|D *(\omega \wedge d u)|^{2} d \mu \\
& +(-1)^{p} \int_{B_{R}\left(x_{0}\right)} \psi\left\langle *(d u \wedge \omega), D^{2} *(d u \wedge * \omega)\right\rangle d \mu \\
& <\epsilon
\end{aligned}
$$

after taking $R$ sufficiently large, $\psi$ as in Lemma 8 and estimating the first term as in [GS] Theorem 7.2. By letting $\psi \rightarrow 1$ we obtain $D^{*}(d u \wedge \omega)=0$ on $\mathcal{R}(u)$. The statement about totally geodesic follows from [C] Theorem 3.3. Q.E.D.

We now treat the higher rank case.

Lemma 10 Let $\tilde{X}=G / K$ be an irreducible symmetric space of noncompact type of rank $\geq 2, \Gamma$ a discrete subgroup of $G$ and $\rho: \Gamma \rightarrow I \operatorname{som}(Y)$. Assume $u: \tilde{X} \rightarrow Y$ is an $\rho$-equivariant harmonic map into a $k$-dimensional $H$ connected complex and $\Gamma$ is a cocompact. Then $u$ is totally geodesic (i.e. $\nabla d u=0)$ in a neighborhood of a regular point.

Proof. The proof is similar to the one for the rank one case, once we establish the Bochner formula of Jost-Yau. Our goal is to verify $[\mathrm{J}]$ Lemma 5.2.1. As in Lemma 9 we will work on the quotient $X=\tilde{X} / \Gamma$ which by assumption is compact. We start with $[\mathrm{J}]$ equation (5.2.4) and note that all terms are tensorial except when we integrate by parts. Let $\psi$ be a nonnegative smooth function vanishing in a small neighborhood of $\mathcal{S}(u)$. We multiply both sides of (5.2.4) by $\psi$, integrate over $X$ and apply integration by parts 
on the first term on the right-hand side. The resulting equation is similar to $[\mathrm{J}]$ equation (5.2.5) except that all integrands get multiplied by the cutoff function $\psi$ and has an extra term

$$
\int_{X}\left\langle\frac{\partial \psi}{\partial \gamma} R_{\beta \alpha \gamma \delta}^{X} u_{x^{\beta}}, u_{x^{\alpha} x^{\beta}}\right\rangle d \mu
$$

on the right-hand side. Since $R_{\beta \alpha \gamma \delta}^{X} u_{x^{\beta}}$ is bounded, this extra term is exactly of the form estimated in Lemma 8 . Hence by taking $\psi \rightarrow 1$, we have verified $[\mathrm{J}]$ equation (5.2.5) and therefore also (5.2.8).

The other point is to justify the formula

$0=\int_{X}|\nabla \nabla u|^{2} d \mu+\int_{X} R_{\alpha \beta}^{X}<u_{x^{\alpha}}, u_{x^{\beta}}>d \mu-\int_{X}<R^{Y}\left(u_{x^{\alpha}}, u_{x^{\beta}}\right) u_{x^{\beta}}, u_{x^{\alpha}}>d \mu$.

To see this, multiply the Eells-Sampson Bochner formula [J] equation (5.2.1) by the cut-off function $\psi$ as above and integrate over $X$. Applying integration by parts on the left-hand side, we obtain a term bounded by

$$
\left.\left|\int_{X}<\nabla \psi, \nabla\right| \nabla u\right|^{2}>d \mu\left|\leq c \int_{X}\right| \nabla \nabla u|| \nabla \psi \mid
$$

where $c$ depends on the Lipschitz constant of $u$. Note that the last integrand above is again of the form estimated in Lemma 8. Hence taking $\psi \rightarrow 1$, we have justfied (8). This verifies $[\mathrm{J}]$ Lemma 5.2.1 which in turn implies that $u$ is totally geodesic (cf. proof of $[\mathrm{J}]$ Theorem 5.3.1). Q.E.D.

Corollary 11 Let $\tilde{X}, Y, \rho$ be as in Theorem 2 and $u: \tilde{X} \rightarrow Y$ a $\rho$ equivariant finite energy harmonic map. Then the image of $u$ must lie in a single DM.

Proof. The first step is to show that if $\gamma:[0,1] \rightarrow \tilde{X}$ is a constant speed parametrization of a geodesic, then $u \circ \gamma$ is a constant speed parametrization of a geodesic in $Y$. Let $x_{0}=\gamma(0)$ and $x_{1}=\gamma(1)$. Let $S$ be a hypersurface perpendicular to $\gamma^{\prime}(1)$ at $x_{1}$. For $r>0$, let $\psi: B_{r}(0) \subset \mathbf{R}^{n-1} \rightarrow S$ be a parametrization of $S$ near $x_{1}$. Define $\Psi: B_{r}(0) \times[0,1] \rightarrow \tilde{X}$ by setting $t \mapsto \Psi(\xi, t)$ to be the constant speed geodesic between $x_{0}$ and $\psi(\xi)$. This map is well-defined and smooth since $\tilde{X}$ has non-positive sectional curvature. In 
fact, its restriction to $B_{r}(0) \times(\epsilon, 1)$ is a diffeomorphism for any $\epsilon$. We claim that there exist $x_{0 i} \rightarrow x_{0}$ and $x_{1 i} \rightarrow x_{1}$ such that if $\gamma_{i}:[0,1] \rightarrow \tilde{X}$ is a constant speed parametrization of a geodesic between $x_{0 i}$ and $x_{1 i}$, then $\gamma_{i}$ maps the open interval $(0,1)$ into $\mathcal{R}(u)$. To prove this claim, we observe that for $\epsilon_{i} \rightarrow 0$, there exists $\xi_{i} \in B_{\epsilon_{i}}(0)$ such that $\left\{\xi_{i}\right\} \times\left(\epsilon_{i}, 1\right) \cap \Psi^{-1}(\mathcal{S}(u))=\emptyset$. Indeed, if $\{\xi\} \times\left(\epsilon_{i}, 1\right) \cap \Psi^{-1}(\mathcal{S}(u)) \neq \emptyset$ for all $\xi \in B_{\epsilon_{i}}(0) \subset \mathbf{R}^{n-1}$, then $\left.\operatorname{dim}_{\mathcal{H}}\left(\Psi^{-1}(\mathcal{S}(u))\right) \cap B_{\epsilon_{i}}(0) \times\left(\epsilon_{i}, 1\right)\right) \geq n-1$; but on the other hand, $\Psi$ restricted to $B_{\epsilon_{i}}(0) \times\left(\epsilon_{i}, 1\right)$ is a diffeomorphism, contradicting the fact that $\left.\operatorname{dim}_{\mathcal{H}}(\mathcal{S}(u))\right) \leq n-2$. This proves the claim by letting $x_{0 i}=\Psi\left(\xi_{i}, \epsilon_{i}\right)$, $x_{1 i}=\Psi_{1}\left(\xi_{i}, 1\right)$ and $\gamma_{i}:[0,1] \rightarrow \tilde{X}$ be defined by $\gamma_{i}(t)=\Psi\left(\xi_{i}, \epsilon_{i}+\left(1-\epsilon_{i}\right) t\right)$. Since $\nabla d u \equiv 0$ in $\mathcal{R}(u)$, we see that $u \circ \gamma_{i}$ is a constant speed parametrization of a geodesic. By the continuity of $u$, this then implies that $u \circ \gamma$ is also.

To complete the proof of the Lemma, it suffices to show that there is no point $x_{0} \in \tilde{X}$ such that $u$ bifurcates into different DM's at $u\left(x_{0}\right)$. Choose an arbitrary point $x_{0} \in \tilde{X}$ and identify $x_{0}=0$ via normal coordinates. Assume without loss of generality that $Y$ is locally isometrically embedded in $\mathbf{R}^{K}, u(0)=0$ and that $T_{u(0)} Y$ is also isometrically embedded in $\mathbf{R}^{K}$. For $\lambda>0$ sufficiently small, define $u_{\lambda}: B_{1}(0) \rightarrow \lambda^{-1} Y$ to be the map $u_{\lambda}(x)=\lambda^{-1} u(\lambda x)$. Since $u$ maps geodesics to geodesics, $u_{\lambda}$ maps geodesics to geodesics of $\lambda^{-1} Y$. From this we can see that $u_{\lambda}$, as a map into $\mathbf{R}^{K}$, is uniformly Lipschitz continuous and converges uniformly on every compact set to a degree 1 homogeneous minimizing map $u_{*}: B_{1}(0) \rightarrow T_{u(0)} Y \subset \mathbf{R}^{K}$. By Proposition 3.1 [GS], $u_{*}$ maps into a $k$-dimensional flat $F$ of $T_{u(0)} Y$. Observe that $\exp _{u(0)} F$ and $u\left(B_{\sigma}(0)\right)$ are both a union of geodesics emanating from $u(0)$. Thus if $u$ bifurcates into different DM's at $x_{0}=0$ (i.e. $u\left(B_{\sigma}(0)\right) \not \subset$ $\exp _{u(0)} F$ for any $\sigma>0$ ), then there exists a geodesic $\gamma$ emanating from 0 such the geodesic $u \circ \gamma$ only intersects $\exp _{u(0)} F$ at $u(0)$. On the other hand since $u_{*}$ is of degree 1

$$
(u \circ \gamma)^{\prime}(0)=u_{*} \circ \gamma^{\prime}(0) \in F
$$

is a nonzero vector and since $u \circ \gamma$ is a geodesic this implies $u \circ \gamma \in \exp _{u(0)} F$, a contradiction. This shows that there exists no point at which $u$ bifurcates into different DM's. Q.E.D.

We now return to the proof of Theorems 2 and 3. Since by assumption there is no invariant unbounded convex closed subset of $Y$ (other than $Y$ 
itself) preserved by $\Gamma, Y$ must be equal to its DM, hence $Y \simeq \mathbf{H}^{k}$. Now again by assumption the image of $u$ must be bounded or equal to $Y \simeq \mathbf{H}^{k}$. In the first case $u$ is constant, whereas the second case means that $u$ is onto hence an isometry (cf. [C]), which is impossible by assumption. This completes the proof of theorems 2 and 3 .

\section{References}

[C] K. Corlette. Archimedian superrigidity and hyperbolic geometry, Ann. Math. 135, 1990, p.165-182.

[DM] G. Daskalopoulos and C. Mese. On the singular set of harmonic maps into DM-complexes, preprint.

[GS] M. Gromov and R. Schoen. Harmonic maps into singular spaces and p-adic superrigidity for lattices in groups of rank one. Publ. Math. IHES 76 (1992) 165-246.

[J] J. Jost. Nonpositive curvature: geometric and analytic aspects. Lectures in Mathematics. ETH Zürich, Birkhäuser Verlag 1997.

[KS1] N. Korevaar and R. Schoen. Sobolev spaces and harmonic maps into metric space targets. Comm. Anal. Geom. 1 (1993) 561-659.

[KS2] N. Korevaar and R. Schoen. Global existence theorem for harmonic maps to non-locally compact spaces. Comm. Anal. Geom. 5 (1997), 333-387.

[M] N. Monod. Superrrgidity for irreducible lattices and geometric splitting. J. AMS 19 (2006), no. 4, 781-814.

[MS] N. Monod and Y. Shalom. Cocycle superrigidity and bounded cohomology for negatively curved spaces. J. Differential Geom. 67 (2004), no. $3,395-455$.

[MMS] I. Mineyev, N. Monod and Y. Shalom. Ideal bicombings for hyperbolic groups and applications. Topology 43 (2004), no. 6, 1319-1344. 